\title{
James D. Frankel, Rectifying God's Name. Liu Zhi's Confucian Translation of Monotheism and Islamic
}

\section{Law}

Honolulu, University of Hawai'i Press, 2011, XXII + 249 p.

Françoise Aubin

\section{OpenEdition \\ Journals}

Édition électronique

URL : http://journals.openedition.org/assr/25478

DOI : $10.4000 /$ assr. 25478

ISSN : $1777-5825$

Éditeur

Éditions de l'EHESS

Édition imprimée

Date de publication : 30 décembre 2013

Pagination : 194

ISSN : 0335-5985

Référence électronique

Françoise Aubin, « James D. Frankel, Rectifying God's Name. Liu Zhi's Confucian Translation of Monotheism and Islamic Law », Archives de sciences sociales des religions [En ligne], 164 | 2013, mis en ligne le 21 février 2014, consulté le 21 septembre 2020. URL : http://journals.openedition.org/assr/ 25478 ; DOI : https://doi.org/10.4000/assr.25478

Ce document a été généré automatiquement le 21 septembre 2020.

(c) Archives de sciences sociales des religions 


\section{James D. Frankel, Rectifying God's} Name. Liu Zhi's Confucian Translation of Monotheism and

\section{Islamic Law}

Honolulu, University of Hawai'i Press, 2011, XXII + 249 p.

Françoise Aubin

\section{RÉFÉRENCE}

James D. Frankel, Rectifying God's Name. Liu Zhi's Confucian Translation of

Monotheism and Islamic Law, Honolulu, University of Hawai'i Press, 2011, XXII + 249 p. 
Curieusement, Liu Zhi (ca. 1660-ca. 1730), un grand penseur et auteur musulman chinois des $\mathrm{XVII}^{\mathrm{e}}$-XVIII ${ }^{\mathrm{e}}$ siècles, est de nos jours très à la mode en milieux sinophone et sinophile. On ne compte plus les articles qui lui sont consacrés dans les revues chinoises spécialisées, qui aiment à démontrer, à juste titre d'ailleurs, que, grâce à la personnalité éminente de Liu Zhi, l'islam est une religion bien chinoise. Et, en Occident, deux ouvrages éblouissants, par Mme Sachiko Murata et son mari William C. Chittick, analysent, à partir des œuvres de l'auteur, le contenu de sa pensée et ses modes d'expression: Chinese Gleams of Sufism (Albany: State Univ. of New York Press, 2000) et surtout le volumineux The Sage Learning of Liu Zhi. Islamic Thought in Confucian Terms (Cambridge, Mass., Harvard Univ. Press,

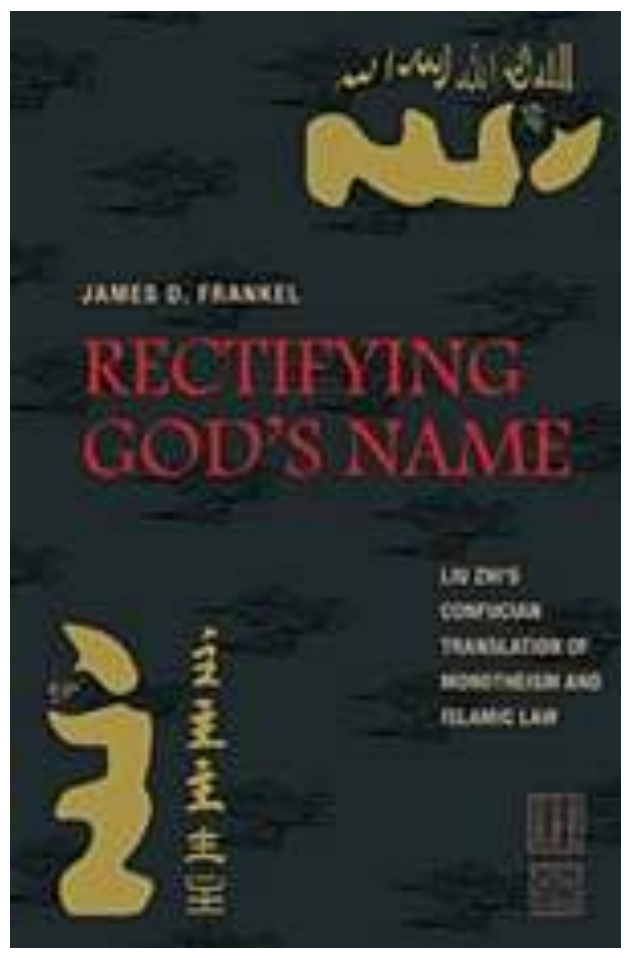
2009).

James Frankel entre donc ici dans un marché déjà bien rempli. Alors que Mme Murata avait choisi, comme base de sa deuxième étude, un grand classique de l'islam chinois, le Tianfang xingli, « Nature et principe en islam », c'est-à-dire une philosophie de l'islam, J. Frankel a judicieusement jeté son dévolu sur l'œuvre que Liu Zhi avait jumelé à son Xingli : le Tianfang dianli [zeyao jie], «[Explications choisies des] Normes et rites de l'islam ». C'est là un ouvrage typique de la littérature dite des han kitāb (han = chinois, $k i t a ̄ b=$ «livre » en arabe), c'est-à-dire des ouvrages musulmans rédigés en chinois par des « ulamā » chinois de haute culture entre le XVII et le XIX ${ }^{\mathrm{e}}$ siècle : le seul han kitāb qui a eu l'honneur d'être inclus dans le grand catalogue de l'empereur Qianlong (r. 1736-1795), le Siku quanshu; le seul han kitāb, selon Frankel, à être uniquement consacré aux rites et aux règles de l'islam (une affirmation un peu exagérée).

L'auteur manie bien le chinois classique et sa connaissance de l'arabe lui permet de placer comme il faut les signes diacritiques dans les transcriptions de cette langue, ce qui est agréable pour le lecteur averti. Hélas! Les a priori favorables tombent dès les premières pages, lorsqu'il apparaît que l'objet déclaré du présent travail, et constamment rappelé de page en page, est l'analyse d'un "syncrétisme » islamochinois. Les connaisseurs de l'islam ne vont pas manquer de sursauter, d'autant plus que notre auteur n'explique pas ce qu'il entend par syncrétisme. Qu'est-ce à dire ? Un islam primitivement pur est-il censé s'être mêlé aux religions chinoises pour donner un « hybride»? Mais alors quel est l'étalon de la pureté originelle supposée ? L'islam en Indonésie ou en Asie centrale est-il aussi un hybride? Absurde de traiter de syncrétiste la plasticité de l'islam pour s'adapter à chaque contexte culturel dans lequel il se trouve plongé.

Le premier chapitre retrace la biographie de Liu Zhi, insistant sur l'ambiance culturelle intense de Nanking, le lieu où il a vécu et qui a été le berceau de plusieurs auteurs musulmans éminents, le choc qu'avait représenté la prise de la ville par les Mandchous 
en juin 1645 et le fait que presque toute la vie littéraire du personnage s'est déroulée sous le règne de Kangxi (r. 1662-1722).

5 Le chapitre 2 traite des traditions littéraires musulmanes en chinois, en arabe et en persan qui l'ont formé, le soufisme qui l'a imprégné n'étant, dit Frankel, que le reflet d'un phénomène universel dans la culture islamique de ce temps. Selon son analyse, le soufisme que l'on est en droit d'attribuer à Liu Zhi, surtout après la lecture de The Sage Learning par Murata et Chittick, ne viendrait que des sources que le penseur musulman ne pouvait éviter de citer: voilà une vue fort politiquement correcte en Chine contemporaine! Ce chapitre sur l'intégration de l'œuvre de Liu Zhi dans la tradition islamique chinoise aurait gagné à être épaulé par une belle thèse parue en allemand mais la bibliographie de J. Frankel n'intègre des titres qu'anglais et chinois: par Barbara Stöcker-Parnian, Jingtang Jiaoyu - die Bücherhallen Erziehung. Entstehung und Entwiklung der islamischen Erziehung in den chinesischen Hui-Gemeinden vom 17.-19. Jahrhundert, Frankfurt am Main, Peter Lang («Europäische Hochschulschriften, Publications Universitaires Européennes, European University Studies ", Reihe XXVII, Asiatische und Afrikanische Studien, Bd 88), 2002 - un travail qui mériterait une traduction anglaise (si Frankel avait consulté des travaux japonais, il aurait aussi pu citer l'étude de Satô Minoru sur les éditions de l'ouvrage de Liu Zhi ici concerné, dans Tôyô gakuhô, 82, nº 3, déc. 2000, p. 57-88). À côté du syncrétisme, le second thème constamment sous-jacent chez Frankel est la supériorité de Liu Zhi, premier et unique parmi les auteurs de han kitāb; mais le lecteur qui a fréquenté les écrits d'autres " ulama $»$ chinois, sans nier les qualités de Liu Zhi, ne peut se retenir de se demander si Frankel a lu autre chose que le Tianfang dianli.

6 Les vues de l'auteur s'affirment au chapitre 3, portant sur les concepts et la terminologie de Liu Zhi. Sans doute la théorie du «syncrétisme » m'avait-elle mise de mauvaise humeur, mais j'ai trouvé l'argumentation lourdement indigeste. Frankel fait un cours, mais on peut se demander à qui il le destine. Sans doute pas à des sinologues, car il ne donne pas les caractères chinois des termes essentiels (une page photocopiée en index final est pourtant à la portée de tous les éditeurs). Le point crucial d'où aurait dû partir la démonstration est que le chinois est une langue sans alphabet, la seule expérience de ce genre qu'ait connue l'islam dans un pays de haute culture (Frankel ne mentionne ce fait essentiel qu'incidemment p. 156-157). À défaut de transcrire, il fallait traduire, et le prétendu syncrétisme est, en fait, une adaptation des concepts et des expressions islamiques en la terminologie usuelle dans la Chine de ce temps : celle du néo-confucianisme - lequel constitue, selon Frankel, un autre exemple de syncrétisme.

Certains concepts fondamentaux, tel sharỉat, ont été effectivement translittérés à l'aide de caractères chinois (la phonétique d'un caractère chinois étant formée d'une consonne suivie d'une voyelle et parfois d'une autre consonne, la restitution du terme originel est souvent hasardeuse), mais le plus souvent les concepts des textes arabes et persans étaient rendus par des termes chinois, néo-confucéens le plus souvent, que le traducteur jugeait équivalents. L'auteur en donne des exemples au chapitre 4, traitant du rituel « comme expression de la simultanéité sino-islamique » et du Tianfang dianli comme manuel ritualiste, sans préciser que les concepts arabes qu'il mentionne sont ses hypothèses personnelles (ou partagées avec Mme Murata). Ainsi il consacre un long développement (p. 105-109) à deux termes arabes «'ibadāt » et « ādāb » en contrepartie $\mathrm{du}$ mot chinois li signifiant «les rites, le rituel, la bienséance ». Si l'on vérifie dans l' Encyclopédie de l'islam ${ }^{2}$ Encyclopaaedia of Islam ${ }^{2} \mathrm{ou}_{\mathrm{II}^{2}}$ - un titre qui ne semble pas faire 
partie des lectures de Frankel-, on voit que 'ibadāt, pluriel de 'ibadā, désigne l'obéissance au maître et la pratique de la religion, c'est-à-dire le rituel de la loi musulmane, donc un équivalent possible pour li. Pour ādāb, pourquoi avoir choisi une forme plurielle (le singulier étant adab) qui désigne à l'époque moderne «la littérature » ? Singulier ou pluriel, une équivalence directe avec li ne parait pas aussi assurée que le prétend l'auteur: le sens originel de adab est «la norme pratique de conduite, la coutume, la sunna ", d'où plus tard un sens dérivé de "bonne éducation, urbanité ", puis de « somme des connaissances » ( $E I^{2}$, vol. I, fasc. 4, 1955, p. 180-181 dans l'éd. franç.).

8 Avec le chapitre 5, nous entrons enfin dans le vif du sujet : « l'esprit du rituel et la lettre de la loi ». Seize des vingt chapitres (juan) du Tianfang dianli sont en effet consacrés, dit l'auteur, à la loi rituelle et à son accomplissement pratique. S'il y a du syncrétisme, c'est bien ici qu'on en doit trouver. Pourtant Frankel reconnait que dans les rites nuptiaux et funéraires les quelques pratiques autochtones qui ont cours sont tout juste marginales, comme cela se voit dans le reste du monde musulman (p. 153). Mais mise à part cette brève remarque, le chapitre est, comme les précédents, tourné vers la terminologie chinoise.

9 C'est ici qu'il faut lire ce qui apparaît être le bon travail sur le Tianfang dianli, celui qui pose correctement les questions et y répond : par Roberta Tontini, «Tianfang dianli : A Chinese Perspective on Islamic Law and its Legal Reasoning ", dans Ming Qing Studies, 2011, p. 491-531 et présentation de l'article par l'éditeur du volume, Paolo Santangelo, un spécialiste du non-conformisme en Chine, p.12-14 (les caractères chinois sont donnés in texto, y compris les nombreuses citations du texte de Liu Zhi; malheureusement les mots arabes manquent de signes diacritiques). Puisque le caractère dian de Tianfang dianli signifie "normes" et que son contenu, ainsi que l'annonce Frankel, est la loi islamique, il faut se placer au niveau du droit et de la légitimité juridique pour juger cet ouvrage de Liu Zhi. C'est précisément ce que fait Tombini, qui commence par exposer la place que tient la tradition légaliste en Chine et celle que le cas chinois propose au débat sur la loi islamique (dian «normes [de la shari’at] ", plutôt que $f a$, «droit basé sur la loi étatique ou la jurisprudence ») dans un milieu politique non musulman - dans l'un et l'autre cas la continuité étant la preuve de la légitimité. Les croyants chinois se situent dans la tradition hanéfite de la jurisprudence sunnite et ils respectent leur souverain, l'empereur mandchou, comme étant la projection de Dieu sur terre ou son ombre. Un musulman ne peut être tenu en esclavage, les femmes ne doivent pas être exclues de l'héritage paternel et si un homme a plusieurs épouses, celles-ci sont toutes du même rang et font l'objet d'un traitement identique, bien que le code Qing alors en vigueur établisse impérativement une hiérarchie entre la première épouse principale et les concubines. Les cinq obligations du croyant, qui concernent l'aspect social de la loi islamique, sont assimilées aux cinq relations sociales du confucianisme, ce qui représente une remarquable stratégie de réinterprétation culturelle, etc. Mais on le voit, aucun « syncrétisme » ne se profile là.

Cette convaincante analyse factuelle rend-elle le travail de Frankel totalement démodé ? Non! D'abord parce que Tombini s'y réfère occasionnellement (sous la forme de la thèse qu'il a soutenue en 2005) ; et surtout parce que l'originalité du chapitre 6, sur "Allah's Chinese Name", rachète les insuffisances des cinq autres chapitres : Liu Zhi a visiblement recherché, pour dénommer Dieu, les solutions adoptées en chinois par les autres traditions monothéistes, c'est-à-dire hébraïsme et christianisme. Ce qui permet 
de mettre à jour les similarités dans la méthodologie et la terminologie des trois traditions abrahamiques transposées en Chine. Voici une façon nouvelle d'aborder l'intégration de l'islam dans la culture chinoise, même si Frankel n'envisage la littérature sino-chrétienne des $\mathrm{XVII}^{\mathrm{e}}-\mathrm{XVIII}{ }^{\mathrm{e}}$ siècles que comme du "syncrétisme » dû à des étrangers (pauvre Ricci!), en omettant les œuvres des convertis chrétiens.

11 Finalement le travail de Frankel souffre de la date de sa publication : 2011, soit deux ans après le travail définitif de Murata et Chittick sur The Sage Learning of Liu Zhi et simultanément à l'étude de Tombini. Serait-il paru plus tôt, on aurait apprécié son originalité. Pour l'heure, il est principalement une étude d'adaptation terminologique de l'arabe en chinois dans une vision de "syncrétisme " qui ne touche en rien le fond $\mathrm{du}$ message religieux. Il est la preuve qu'une bonne thèse de $\mathrm{PhD}$ ne donne pas forcément un succès de librairie. 